\title{
Methods for Linear Radial Motion Estimation in Time-of-Flight Range Imaging
}

\author{
Lee Streeter \\ School of Engineering, University of Waikato, Gate 1, Knighton Rd. Private Bag 3105, \\ Hamilton 3240, New Zealand.
}

\begin{abstract}
Motion artefacts in time-of-flight range imaging are treated as a feature to measure. Methods for measuring linear radial velocity from range imaging cameras are developed and tested. With the measurement of velocity, the range to the position of the target object at the start of the data acquisition period is computed, effectively correcting the motion error. A new phase based pseudo-quadrature method designed for low speed measurement measures radial velocity up to $\pm 1.8 \mathrm{~m} / \mathrm{s}$ with RMSE $0.045 \mathrm{~m} / \mathrm{s}$ and standard deviation of $0.09-0.33 \mathrm{~m} / \mathrm{s}$, and new high-speed Doppler extraction method measures radial velocity up to $\pm 40 \mathrm{~m} / \mathrm{s}$ with standard deviation better than $1 \mathrm{~m} / \mathrm{s}$ and RMSE of $3.5 \mathrm{~m} / \mathrm{s}$.
\end{abstract}

Keywords: Time-of-flight range imaging, linear radial motion estimation.

\section{INTRODUCTION}

Time-of-flight (ToF) range imaging is an optical lidar method for full field measurement of distance. ${ }^{1}$ A ToF camera is an active imaging system that uses amplitude modulated continuous wave (AMCW) illumination in homodyne with a sensor demodulation signal. The camera encodes distance by the ToF of light as the phase offset between light return and sensor waveforms, which directly influences the integrated intensity of light in a single camera frame. However, in addition to the ToF of the light, the amplitude of the light return, and background light, both also affect the integrated intensity, so there are three unknowns, and at least three frames must be captured before we can compute the distance. The frames are taken in sequence by a single camera where it is assumed that the scene is static during the capture process, and herein lies the problem of motion in ToF range imaging, that motion violates this static scene assumption and causes error in the distance measurements. But, motion can also be treated as another variable in ToF imaging, especially radial motion which manifests as a Doppler shift. ${ }^{2,3}$ This work is concerned with the measurement of linear radial motion (linear velocity towards or away from the camera aperture), and mitigation of error due to radial motion in measurements of distance.

Radial motion estimation in ToF cameras by modified camera operation methods leverage special features of heterodyne modulation ${ }^{3}$ and continuous wave modulation. ${ }^{2}$ These successful methods, however, do not operate the ToF cameras in standard off-the-shelf modes. In earlier work, modelling led to a modified range reconstruction method that reduced the impact of radial motion. ${ }^{4}$

Radial motion is just one form that affects ToF imaging. The other is transverse motion that is orthogonal to the radial axis (in spherical coordinates). Two major approaches dominate the literature for transverse motion. The first major approach is estimation of transverse motion and subsequent correction. ${ }^{4-6}$ The second major approach follows two steps: the first step the examination the raw data from the ToF camera for inconsistencies; and the second step is computation of the distance based on the remaining consistent data. ${ }^{7,8}$ Concordant with the second major approach, hardware control of integration times also reduces the impact of motion. ${ }^{9}$ Shortening the integration time (or using fewer raw frames) reduces the information comprising the distance measurement, increasing the overall noise. Hence, we take the motion measurement route, where large motion effects are encouraged.

Further author information: (Send correspondence to L.S.)

E-mail: streeter@waikato.ac.nz, Telephone: +64 78384106. 
In this work we derive and test two new methods for estimating linear radial velocity. The first method is based a pseudo-quadrature modulation method that exposes radial motion as an extra phase shift, under the assumption that the speed is small. The second method invokes principles of times series analysis of noisy data, and transforms the radial motion problem into a correlation problem, suitable for regression analysis. In Section 2 we review ToF camera operational theory, extend that theory to include radial linear motion, and derive new methods to measure radial motion. In Section 3 we describe the experiments to text the new methods, and in Section 4 present the results and discuss the findings.

\section{THEORY}

We begin by outlining the theory behind standard AMCW ToF homodyne range imaging method ${ }^{1}$ and the effect that linear radial motion has. Based on this theory, we then derive the new methods of computing radial velocity measurement from the data available from a ToF camera.

The active illumination of a ToF range imaging system is continuously amplitude modulated according to the modulation signal. The illumination source is typically a light emitting diode or diode laser, which respectively can typically be modulated in the $10 \mathrm{~s} \mathrm{MHz}$ and 100s MHz. The light emitted by the source is backscattered by the scene and returns to the camera sensor. Let $f$ be the modulation frequency of the camera, $d$ the camera to scene distance, and $c$ the speed of light in air, then light return is phase shifted by $\phi$, viz.

$$
\phi=\frac{4 \pi d f}{c},
$$

where the factor of four contains a factor of two due to the light travelling the distance, $d$, twice. The light return, $r(t ; \phi)$, and sensor, $s(t ; \theta)$, modulations are idealised as a sine waves, which are modelled as

$$
r(t ; \phi)=A \sin (2 \pi t+\phi)+B, \quad s(t ; \theta)=\frac{1}{2} \sin (2 \pi t-\theta)+\frac{1}{2} .
$$

where $A$ is the return amplitude, $B$ is the background light level, and $\theta$ is an electronic phase offset between the light source and sensor. The light return and demodulation signals mix at the sensor, which integrates over time $T$ viz.

$$
I(\theta ; \phi)=\int_{t=0}^{T} r(t, \phi) s(t ; \theta) d t=\alpha \cos (\phi+\theta)+\beta,
$$

where $\alpha$ and $\beta$ are constants that depend on $A, B$, and $T$. The camera effectively takes the correlation between $r(t ; \phi)$ and $s(t ; \theta)$, hence, Eqn. (3) is called the correlation wavefunction. ToF cameras are designed so that $\theta$ is adjustable to discrete values, $\theta=\theta_{n}, n=1 \ldots N$, between integrations, and in doing such adjustment we sample $I_{n}=I\left(\theta_{n} ; \phi\right)$. We refer to the $I_{n}$ as raw frames.

Linear radial motion, or linear motion along the radial axis (in terms of spherical coordinates) causes a Doppler shift, or stretching, of the correlation wavefunction. This shift is most practically modelled by the inclusion of the velocity parameter $k$, where $0<k$, viz.

$$
I_{n}=\alpha \cos \left(\phi+k \theta_{n}\right)+\beta .
$$

Estimating $k$ from the $I_{n}$, therefore, gives a measurement of the radial linear motion. But, bandwidth limitations of data transmission and the desire to measure distance at full frame rates means that the $\theta_{n}$ used are small in number, therefore estimating $k$ is more challenging than in other contexts such as Doppler RADAR ${ }^{10}$ or Ultrasound. ${ }^{11}$ In the remainder of this section we present two new methods for estimating $k$ from the $I_{n}$, and extend the estimation of $\phi$ to account for radial motion.

\subsection{Pseudo-Quadrature Signal Analysis Velocity Estimation}

The first insight that leads to estimation of $k$ is, using Euler's famous equation, ${ }^{12}$ that we may rewrite $I_{n}$ as

$$
I_{n}=\frac{1}{2}\left(P \tau^{n}+\bar{P} \tau^{-n}\right)+\beta
$$


where $i$ is the imaginary unit, $P=e^{i \phi}, \tau=e^{-i k \Delta \theta}$, we choose the phase shift size $\Delta \theta$, hence phase steps $\theta_{n}=n \Delta \theta$, and $\bar{P}$ denotes the complex conjugate of $P$. Taking a second raw frame with a $\pi / 2$ radian shift from $I_{n}$ we have

$$
\begin{aligned}
I_{n}^{\prime}=\alpha \cos \left(\phi+k\left(\theta_{n}+\pi\right)\right)+\beta=\alpha \sin \left(\phi+k \theta_{n}+(k-1)\right. & \pi)+\beta \\
& =\frac{-i}{2}\left(P \tau^{n} e^{i(k-1) \pi / 2}-\bar{P} \tau^{-n} e^{-i(k-1) \pi / 2}\right)+\beta .
\end{aligned}
$$

Making the assumption that $|k-1|$ is small then after the acquisition of the raw frames

$$
m_{n}=I_{n}+i I_{n}^{\prime} \approx P \tau^{n}+(1+i) \beta .
$$

With three $m_{n}, n=0,1,2$, in hand, it follows that

$$
\frac{m_{2}-m_{1}}{m_{1}-m_{0}}=\frac{P \tau^{2}-P \tau^{1}}{P \tau^{1}-P \tau^{0}}=\tau \frac{P(\tau-1)}{P(\tau-1)}=\tau .
$$

It is straightforward to recover the velocity parameter $k$ from $\tau$ with the use of the usual trigonometric operation

$$
k=\frac{1}{\Delta \theta} \tan ^{-1}\left(\frac{\mathcal{I}(\tau)}{\mathcal{R}(\tau)}\right)
$$

Where $\mathcal{I}(\tau)$ and $\mathcal{R}(t)$ are, respectively, the imaginary and real parts of $\tau$. With $\tau$ in hand, we compute $P$ as

$$
\frac{m_{2}-m_{1}}{\tau^{2}-\tau^{1}}=P \frac{\tau^{2}-\tau^{1}}{\tau^{2}-\tau^{1}}=P
$$

Like above, it is straightforward to recover $\phi$ from $P$.

This is a quadrature analysis method, but there is a small time delay in the acquisition of each $I_{n}$ and the corresponding $I_{n}^{\prime}$. Therefore this is a pseudo-quadrature signal analysis (PQSA) technique of velocity estimation. The small speed approximation made in the above analysis limits the applicability of PQSA to low velocities, such as those found in indoor environments. For faster motion we need a method that extracts $k$ from the $I_{n}$ with no low speed assumptions, which is the topic of the next section.

\subsection{Correlation Analysis Velocity Estimation}

The second insight leading to measurement of $k$ from the $I_{n}$ is that calculus operations bring coefficients out of trigonometric functions. Specifically, applying indefinite integration and differentiation of $I_{n}$ with respect to $\theta$ exposes the rate of change, which is the parameter we wish to measure. Moreover, invoking the principles of stochastic calculus, ${ }^{13}$ we have assurances that we can view the $I_{n}$ as a noisy time series and perform the necessary calculus operations. Stochastic calculus, as the name suggests, takes a statistical view of time series data, leaverageable for parameter estimation.

We begin by extending the correlation wavefunction model to include random noise

$$
I_{n}=\alpha \cos \left(\phi+k \theta_{n}\right)+\beta+\sigma \epsilon_{n},
$$

where the $\epsilon_{n}$ are samples from a Gaussian distribution of mean zero and variance 1, and $\sigma$ is some positive constant that describes the standard deviation of the noise in $I_{n}$. From discrete stochastic calculus we can approximate the integral and derivative of $I_{n}$ with a discrete cumulative sum and discrete difference

$$
\begin{aligned}
J_{n} & =\sum_{m=0}^{n} I_{m} \approx \frac{\alpha}{\Delta \theta k} \sin \left(\phi+k \theta_{n}\right)+\beta(n+1)-\frac{\alpha}{\Delta \theta k} \sin (\phi)+\sum_{m=0}^{n} \epsilon_{m}, \\
D_{n} & =I_{n+1}-I_{n} \approx-\Delta \theta k \alpha \sin \left(\phi+k \theta_{n}\right)+\epsilon_{n+1}-\epsilon_{n} .
\end{aligned}
$$




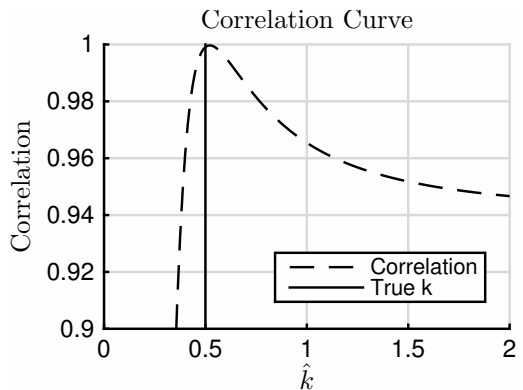

(a) Pearson's correlation, $k=0.5$.

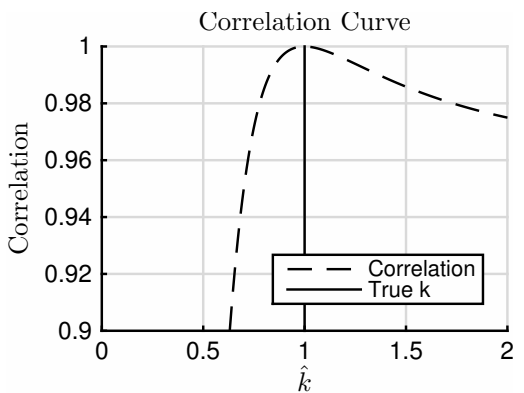

(b) Pearson's correlation, $k=1.0$.

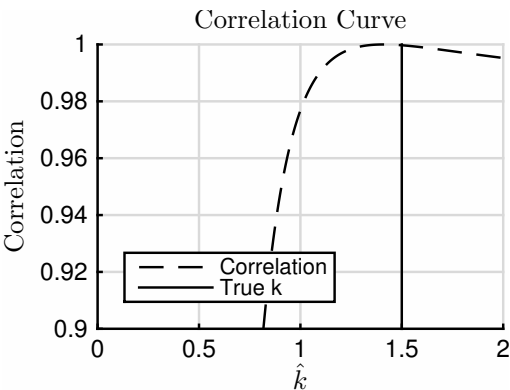

(c) Pearson's correlation, $k=1.5$.

Figure 1: Simulation examples of Pearson's correlation coefficient between $\Omega(n ; \hat{k})$ and $\theta_{n}$.

Both $J_{n}$ and $D_{n}$ have sine terms, but $J_{n}$ has the second term that is linear with $n$. Therefore, we form the objective function

$$
\Omega(n ; \hat{k})=J_{n}+\hat{k} D_{n}=-\frac{\alpha}{\Delta \theta k} \sin (\phi)+(1+n) \beta+\left(\frac{1}{\Delta \theta k}-\hat{k} \Delta \theta k\right) \alpha \sin \left(\phi+k \theta_{n}\right)+\epsilon_{n+1}-\epsilon_{n}+\sum_{m=0}^{n} \epsilon_{m}
$$

When $\hat{k}=1 /(\Delta \theta k)^{2}$, the coefficient of sine in the third term of the right hand side of Eqn. (13) vanishes and $\Omega(n ; \hat{k})$ becomes a function of points on a straight line. The same value of $\hat{k}$ will also maximise Pearson's correlation coefficient ${ }^{14}$ between $\Omega(n ; \hat{k})$ and $n$. In Fig. 1 we give examples of the correlation coefficient as a function of $\hat{k}$. The peak of the curve follows the true value of $k$. Therefore, finding the peak of the correlation coefficient is a practical estimator of $\hat{k}$, albeit an inefficient one. We call this method correlation analysis velocity estimation (CAVE).

Reexamining $J_{n}$ and $D_{n}$ we see that, for the optimal value of $\hat{k}$, we may write

$$
J_{n}+\hat{k} D_{n}=c_{1} n+c_{0}
$$

where $c_{1}$ and $c_{0}$ are, respectively, coefficients that also account for the linear and constant parts of $\Omega(n ; \hat{k})$. Therefore, an efficient means of finding the optimal value of $\hat{k}$ is to use linear regression to indirectly perform correlation analysis by solving the following linear system

$$
\left[\begin{array}{c}
J_{1} \\
\vdots \\
J_{N}
\end{array}\right]=\left[\begin{array}{ccc}
-D_{1} & 1 & 1 \\
\vdots & \vdots & \vdots \\
-D_{N} & N & 1
\end{array}\right]\left[\begin{array}{c}
\hat{k} \\
c_{1} \\
c_{0}
\end{array}\right]
$$

The number of columns of the matrix in Eqn. (15) is fixed to three, therefore solving Eqn. (15) by QR decomposition has efficiency $O(N)$. However, it should be noted that there is a fixed number of $3^{3}$ operations included in the matrix inversion.

Simulations of CAVE by linear regression are shown in Fig. 2 with $N=9$. Both idealised pure cosine correlation waveform and the correlation waveform with the third and fifth order harmonics of square wave modulation are simulated. The harmonic error in $\hat{k}$ increases with velocity towards the camera, ostensibly occurring as the portion of the correlation waveform that is sampled reduces. The exact mechanism for the increase of error is not precisely understood, and left for future work.

\subsection{Estimation of Phase}

The estimation of phase is inherent to PQSA. For simple DoP the phase is estimated before the radial velocity is computed. For CAVE we need to return to the correlation waveform. Expanding the cosine in Eqn. (4) gives. ${ }^{15}$

$$
I_{n}=A \cos (\phi) \cos \left(k \theta_{n}\right)-A \sin (\phi) \sin \left(k \theta_{n}\right)+\beta .
$$




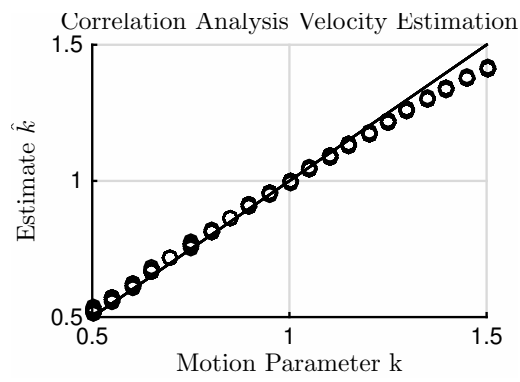

(a) Simulation of CAVE with pure cosine correlation wavefunctions.

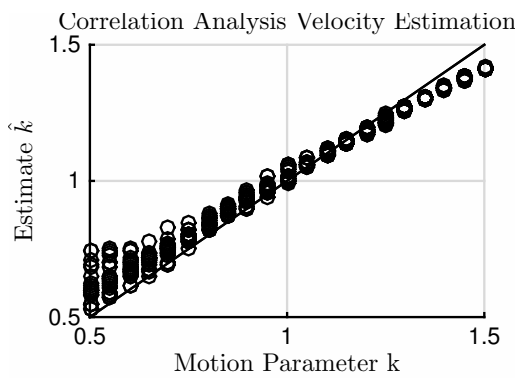

(b) Simulation of CAVE with the first, third, and fifth harmonics.

Figure 2: Simulation of CAVE. Motion towards (away from) the camera corresponds to $k<1(k>1)$.

Eqn. (16) can be factorised to form the matrix product

$$
I_{n}=\left[\cos \left(k \theta_{n}\right)-\sin \left(k \theta_{n}\right) 1\right]\left[\begin{array}{c}
\mathcal{R}(P) \\
\mathcal{I}(P) \\
\beta
\end{array}\right],
$$

where $\mathcal{R}(P)$ and $\mathcal{I}(P)$ are respectively the real and imaginary parts of $P$. Once $k$ is measured, it is straightforward to write the $N$ equations for the $I_{n}$ as a linear system and find $P$ and $\beta$, where $P=\mathcal{R}(P)+i \mathcal{I}(P)$.

\section{MATERIALS AND METHODS}

We test the two radial motion measurement methods given. We also compare to motion measurement by simply taking the difference of phase (DoP) between consecutive range images.

The data are captured with a proprietary prototype camera on which we can control the phase steps and modulation frequency. The modulation frequency of the camera is set to $70 \mathrm{MHz}$, giving an ambiguity distance of $15 / 7=2.143 \mathrm{~m}$. All camera and translation stage control and synchronisation, and processing of data, is done using Matlab (The Mathworks, Natick, MA, USA) on a i3 Windows 7 based PC with $8 \mathrm{G}$ of ram.

A white board target is affixed to a Macron 6 translation stage (Macron Dynamics, Croydon, PA, USA) that is $3.4 \mathrm{~m}$ in length and fitted with a stepping motor capable of translating objects up to $2.4 \mathrm{~m} / \mathrm{s}$. These speeds are suitable for live low speed tests of PQSA. To test CAVE, $N=9$ phase steps is chosen spread evenly over $3 \pi$ radians, hence $\Delta \theta=\pi / 3$. To test speeds of up to $40 \mathrm{~m} / \mathrm{s}$, full nine phase step sets of raw frames are acquired of the board at static positions set by the translation stage, and then semi-simulated $N=9$ phase step sets of raw frames are constructed in the computer. The initial positions are $1.990 \mathrm{~m}$ for motion away from the camera, and $3.190 \mathrm{~m}$ for motion towards the camera. The phase is computed for no motion at the initial positions, and motion without correction; and after measurement of the radial velocity by CAVE the phase is computed with motion correction. The mean and standard deviation of the velocity measurements are examined over an $11 \times 11$ pixel region of interest.

In another experiment we use a MESA Imaging SR4000 camera (Heptagon Micro Optics, Singapore) to demonstrate velocity estimation by a modified form of PQSA. The SR400 is restricted to the classical four quad data acquisition method, viz. $\theta_{n} \in\{0, \pi / 2, \pi, 3 \pi / 2\}$. To perform velocity estimation using the SR4000, we take data from three separate sets of four quad raw frames and compute $m_{0}$ from a first set of $N$ raw frames, $m_{1}$ from second set of raw frames, and $m_{2}$ from the third. Thus, we devise a multi-set extension of PQSA. The relative effect of the phase shift in $\tau$ is larger between the $m_{i}$ than between $I_{n}$ and $I_{n}^{\prime}$, which is conjectured to improve the linearity of the velocity measurements and reduce the apparent noise level in the velocity measurements.

\section{RESULTS AND DISCUSSION}

In Figs. 3a-3c the velocity measurements from the live low speed tests are displayed and compared. The real time PQSA results are of the direct implementation of the method of Section 2.1 where the multi-set pseudo-quadrature 


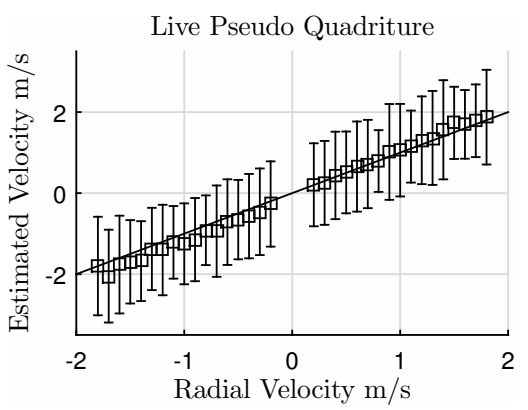

(a) Real time PQSA velocity estimation.

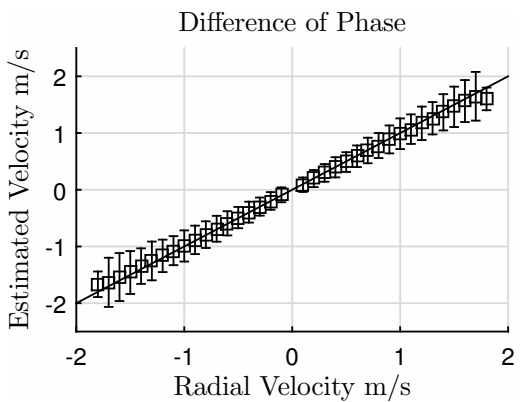

(c) Radial velocity measurement by simple DoP.

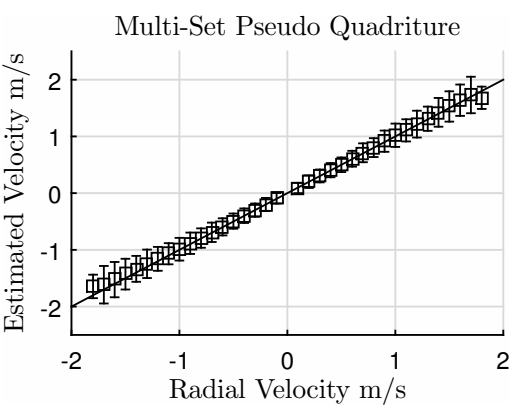

(b) PQSA velocity estimation over multiple sets.

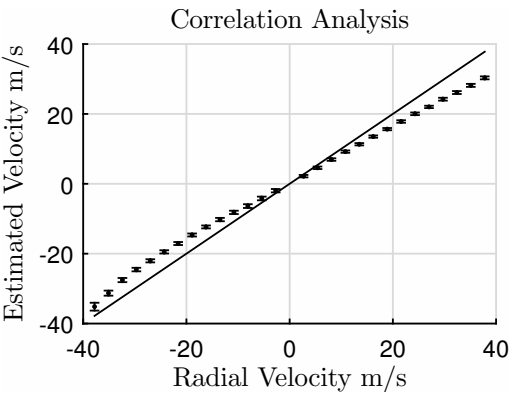

(d) The CAVE high-speed results showing shape due to harmonics.

Figure 3: Velocity measurement tests. Error bars represent one standard deviation. The graphs 3a-3c are low speed live velocity measurement tests by analysis of phase. Multi-set PQSA increases the displacement between frames, reducing the apparent noise level in the velocity estimates. The high speed semi-simulated test result is in $3 \mathrm{~d}$.

results are over multiple sets of raw frames taken with the SR4000 camera. The standard deviation is reduced by one third or better between PQSA and multi-set PQSA, see Table 1, confirming the conjecture that the greater motion between raw frame sets than between raw frames leads to less noise in the velocity measurements. The measurements are all within uncertainty of the reference velocity, so we can make no statement of the relative linearity between PQSA and multi-set PQSA. Therefore, the conjecture that multi-set PSQA improves linearity is not confirmed. In future work faster object motion will be emplyed to test this linearity conjecture. The noise level of multi-set PSQA is slightly less than DoP, Table 1. In these tests six phase steps are used for PSQA and eight for DoP (four per phase measurement). DoP by six phase steps is possible (three per phase measurement), but at an increase in noise.

Table 1: Summary of Methods for Linear Radial Velocity Measurement. PQSA is pseudo-quadrature signal analysis, PQSA multi. is over multiple phase step sets, CAVE is correlation analysis velocity estimation, and DoP is simple difference of phase. NR in prior literature denotes not reported.

\begin{tabular}{c|ccc}
\hline & & & \\
& Max. speed tested $\mathrm{m} / \mathrm{s}$ & $\mathrm{STD} \mathrm{m} / \mathrm{s}$ & $\mathrm{RMSE}$ of mean $\mathrm{m} / \mathrm{s}$ \\
\hline & & & \\
DoP & 1.8 & $0.13-0.43$ & 0.049 \\
PQSA & 1.8 & $\approx 1$ & 0.15 \\
Multi. PQSA & 1.8 & $0.09-0.33$ & 0.045 \\
CAVE & 40 & $<1$ & 3.5 \\
Heide et al. $^{3}$ & 6 & $\leq 0.02$ & $\mathrm{NR}$ \\
Whyte et al. $^{2}$ & 1.1 & $0.003-0.034$ & $\mathrm{NR}\left(R^{2}=0.9969\right)$
\end{tabular}

The semi-simulated high speed test of CAVE is presented in Fig. 3d. CAVE has noise STD better than $1 \mathrm{~m} / \mathrm{s}$ 
in this test. As summarised in Table 1, the accuracy as measured by the RMSE is $3.5 \mathrm{~m} / \mathrm{s}$, and the measured velocities deviate from the true value beyond uncertainty. Harmonic error leads to the bias seen in Fig. $3 \mathrm{~d}$ in that the velocity measurements deviate from the true radial velocity beyond uncertainty.

In comparison, $\mathrm{CW}$ homodyne modulation ${ }^{2}$ measured velocity only up to $1.1 \mathrm{~m} / \mathrm{s}$ with standard deviations in the order of $3 \%$ of the speed, albeit requiring more than 100 raw measurements. Heterodyne and homodyne modulation together ${ }^{3}$ with two cameras notably measured radial velocity up to $\pm 20 \mathrm{~m} / \mathrm{s}$ simulated for calibration and up to $6 \mathrm{~m} / \mathrm{s}$ testing with reported errors less than $0.2 \mathrm{~m} / \mathrm{s}$, but with significant modification to the camera hardware. Taking three frames across the two cameras, and leveraging background subtraction on pixel, they measured velocity and distance simultaneously.

CAVE is extensible to polynomial motion. Examining Eqns. 14-15, acceleration is accounted for by including a second order term $n^{2}$. This process is more subtle than simply adding an extra column to the matrix in Eqn. (15), however, hence is currently untested and left for future work. Also, in future work, live controlled high speed motion systems will be investigated for testing of the motion measurement methods, including nonlinear motion.

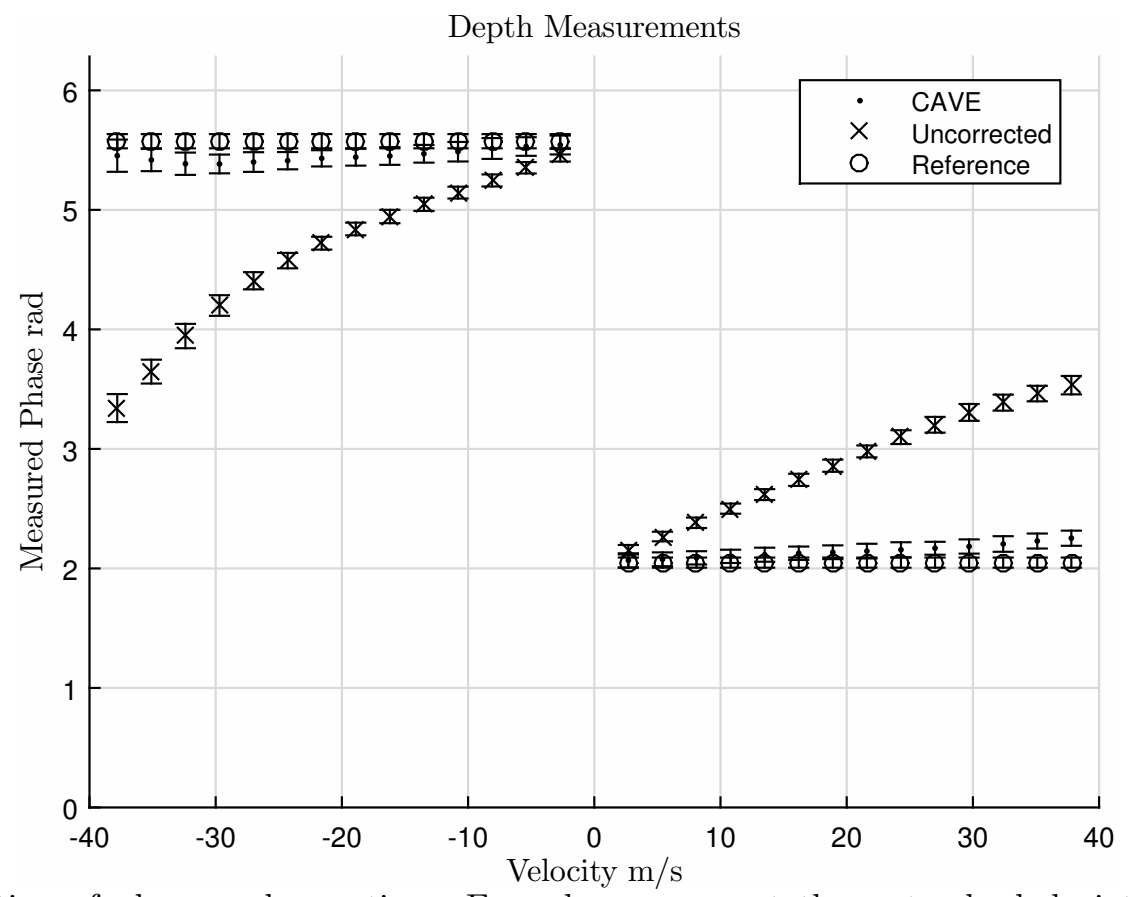

Figure 4: Estimation of phase under motion. Error bars represent three standard deviations. The CAVE measurement corrected phase computation is improved over no motion correction, albeit at an increase in STD.

Phase estimate accuracy and standard deviation are summarised in Fig. 4. Using the CAVE measurements, phase estimation by solving Eqn. (17) reduces the error on average, but the STD in phase estimates increases on average from 17 millirad to 24 millirad, i.e. a factor of 1.4 .

\section{CONCLUSION}

In this work two new methods for linear radial velocity measurement from time-of-flight range image camera data are presented, namely CAVE and PQSA. The new methods are tested and compared to previous methods, totalling six distinct approaches to measuring radial motion. Correction of motion error in the phase measurements is also tested, along with the impact of radial motion correction on the standard deviation of phase measurement. Overall, CAVE performs well on high speed test with standard deviation of less than $1 \mathrm{~m} / \mathrm{s}$ and RMSE of 3.5 $\mathrm{m} / \mathrm{s}$ over range $(-) 40-(+) 40 \mathrm{~m} / \mathrm{s}$. An extension of PQSA, called multi-set PQSA performs well at low speeds with standard deviations of $0.09-0.33 \mathrm{~m} / \mathrm{s}$ and RMSE of $0.045 \mathrm{~m} / \mathrm{s}$ over the range $(-) 1.8-(+) 1.8 \mathrm{~m} / \mathrm{s}$. 
The results show that through careful modelling radial motion is measurable and the effect of such motion correctable. We do not need to reduce the number of frames, nor the integration time of each raw frame, to reduce the influence radial motion has on the quality of the data. Instead, motion artefacts are embraced as something to be measured, rather than mitigated, in which case it is advantageous to increase the total integration time to increase the impact, hence measurability of motion. Future work will extend these principles to the discontinuous motion artefacts cause by an edge passing transverse to the radial axis over the view of a pixel. We are currently investigating acceleration measurement and statistical methods for transverse motion measurement and correction. The ultimate goal is to extend the ideas presented herein to full three-dimensional velocity measurement.

\section{ACKNOWLEDGEMENTS}

This work was funded by Marsden Council Fast Start grant UOW1501. The author acknowledges useful discussion with John Godbaz in the early stages of this research.

\section{REFERENCES}

[1] Lefloch, D., Nair, R., Lenzen, F., Schäfer, H., Streeter, L., Cree, M. J., Koch, R., and Kolb, A., [Technical Foundation and Calibration Methods for Time-of-Flight Cameras], 3-24, Springer Berlin Heidelberg, Berlin, Heidelberg (2013).

[2] Whyte, R., Streeter, L., Cree, M. J., and Dorrington, A. A., "Application of lidar techniques to time-of-flight range imaging," Applied Optics 54, 9654-9664 (Nov 2015).

[3] Heide, F., Heidrich, W., Hullin, M., and Wetzstein, G., "Doppler time-of-flight imaging," Transactions on Graphics 34(4), 36:2-36:11 (2015).

[4] Lindner, M. and Kolb, A., "Compensation of motion artifacts for time-of-flight cameras," in [Dynamic 3D Vision Workshop], 5742, 16-27 (September 2009).

[5] Lottner, O., Sluiter, A., Hartmann, K., and Weihs, W., "Movement artefacts in range images of time-of-flight cameras," in [International Symposium on Signals, Circuits and Systems], 117-120 (July 2007).

[6] Streeter, L. and Dorrington, A. A., "Coded exposure correction of transverse motion in full-field range imaging," Optical Engineering 53(10), 102109-1-102109-10 (2014).

[7] Lee, S., "Time-of-flight depth camera motion blur detection and deblurring," IEEE Signal Processing Letters 21(6), 663-666 (2014).

[8] Hussmann, S., Hermanski, A., and Edeler, T., "Real-time motion artifact suppression in tof camera systems," IEEE Transactions on Instrumentation and Measurement 60(5), 1682-1690 (2011).

[9] Buettgen, B. and Oggier, T., "Demodulation sensor with separate pixel and storage arrays," (Sept. 13 2016). US Patent 9,442,196.

[10] Skolnik, M., [Radar Handbook], McGraw Hill, NY, USA, third ed. (2008).

[11] Bonnefous, O. and Pesqué., P., "Time domain formulation of pulse-doppler ultrasound and blood velocity estimation by cross correlation," Ultrasonic Imaging 8(2), 73-85 (1986).

[12] Streeter, L. and Dorrington, A. A., "Simple harmonic error cancellation in time of flight range imaging," Optics Letters 40(22), 5391-5394 (2015).

[13] Klebaner, F. C., [Introduction to Stochastic Calculus with Applications], Imperial college Press, London, UK, third ed. (2012).

[14] Freund, R. J., Wilson, W. J., and Sa, P., [Regression Analysis Statistical Modelling of a Response Variable], Elsevier Science, Amsterdam, Netherlands, second ed. (2006).

[15] Streeter, L., Cree, M. J., and Dorrington, A. A., "A strategy for the correction of effects of jitter in AMCW lidar images," in [The 28th International Conference of Image and Vision Computing New Zealand], 500-505 (2013). 